\title{
Comparative evaluation of coagulase-negative staphylococci (CoNS) adherence to acrylic by a static method and a parallel-plate flow dynamic method
}

\author{
Nuno Cerca ${ }^{\mathrm{a}}$, Gerald B. Pier ${ }^{\mathrm{b}}$, Rosário Oliveira ${ }^{\mathrm{a}}$, Joana Azeredo ${ }^{\mathrm{b}, *}$ \\ ${ }^{a}$ Centro de Engenharia Biológica, Universidade do Minho, Campus de Gualtar, 4710-057 Braga, Portugal \\ b Channing Laboratory, Department of Medicine, Brigham and Women's Hospital, Harvard Medical School, Boston, MA, USA \\ Received 11 June 2004; accepted 14 June 2004 \\ Available online 10 August 2004
}

\begin{abstract}
The main goal of this work was to evaluate the adhesion to acrylic of several clinical strains of Staphylococcus epidermidis and Staphylococcus haemolyticus using both static and dynamic adhesion methods, and to compare the results obtained with these two methods. Adhesion was evaluated using the static slide method with different washing procedures, and the parallel plate flow chamber method. The extent of S. epidermidis adhesion, assessed by both methods, was greater than that of $S$. haemolyticus. The number of bacteria which adhered using the static method was lower than that using the dynamic method. It was found that the simple static method, when performed with an accurate washing procedure, can be as effective as the dynamic flow method for assessing differences in the adherence capacity of strains. Although the dynamic flow method yielded more overall information, its greater complexity and cost may not always justify its use for certain experimental comparisons. This investigation has shown that simple static adhesion methods, when performed accurately, can be used to evaluate differences in adhesion capacity.
\end{abstract}

(c) 2004 Elsevier SAS. All rights reserved.

Keywords: Adhesion; Parallel flow plate; Slide static method; Staphylococcus epidermidis; Staphylococcus haemolyticus

\section{Introduction}

Healthy human skin and mucous membranes represent the natural habitat of coagulase-negative staphylococci (CoNS) such as Staphylococcus epidermidis and Staphylococcus haemolyticus [25]. In predisposed hosts, usually with an indwelling medical device, CoNS have become significant nosocomial pathogens [14]. The major virulence factor associated with this organism's ability to cause infections is dependent upon adhesion to medical devices and formation of a biofilm [25].

Acrylic is a polymer often used in the manufacturing of medical implants like bone-cement, intraocular artificial lenses and cranioplastic implants [10]; adhesion to acrylic

\footnotetext{
${ }^{*}$ Corresponding author.

E-mail address: jazeredo@deb.uminho.pt (J. Azeredo).
}

surfaces may contribute to serious clinical consequences such as persistent and chronic infections [20].

Microbial adhesion to inert surfaces such as acrylic has been shown to be a complex process, involving physicochemical interactions between the polymer and protein and polysaccharide factors of the bacteria $[3,13,24]$. In addition, a wide variety of experimental systems have been developed to study microbial adhesion to inert surfaces, but there is no consensus as to which is most representative of the infectious process in a human.

One of the simplest methods for studying bacterial adhesion is the static adherence method [5]. In this method, a slide or similar piece of substratum such as a catheter is immersed in a microbial suspension and a batch adhesion process is allowed to occur. After exposure, the substratum is washed for removal of non-adherent bacteria and then adhering bacteria can be enumerated in situ. More elaborate methods are available for studying microbial adhesion, one 
of which, the parallel-plate flow chamber, is considered by many investigators to have important advantages [5]. With the parallel-plate flow chamber, adhesion to surfaces can be studied in a more controlled hydrodynamic environment, and more experimental parameters can be measured, such as the initial adhesion rate or the removal rate after passage of an air-liquid interface [6].

According to some authors this type of system provides a more accurate evaluation of bacterial adhesion than the static adhesion system because of the washing step required in the latter method [5]. This washing procedure, necessary for removing non- or loosely adhering cells, strongly influences the adhesion results. Although it is a crucial step in static adhesion assays most of the reported data resulted from adhesion assays performed under uncontrolled washing procedures.

In this study the adhesion to acrylic of 7 CoNS strains was evaluated by static slide methods, in which different washing steps, carefully controlled, were performed, as well as by the dynamic parallel-plate flow chamber. A comparison of the results obtained by these methods was performed.

\section{Material and methods}

\subsection{Bacterial strains}

In this work, 5 S. epidermidis strains and $2 S$. haemolyticus strains were used. S. epidermidis 9142 is a known producer of the major surface polysaccharide promoting CoNS adherence and biofilm formation, referred to as either polysaccharide intercellular adhesin (PIA) or by its chemical composition, poly- $N$-acetyl glucosamine (PNAG) and S. epidermidis $9142-\mathrm{M} 10$ is a strain isogenic to 9142 that contains a transposon inserted into the ica locus which encodes the biosynthetic enzymes for producing PIA/PNAG and thus does not produce this molecule. S. epidermidis IE75, S. epidermidis IE186 and S. haemolyticus IE246 were isolated from infective endocarditis patients; S. haemolyticus M176 and S. epidermidis M187 were isolated from patients with peritonitis associated with renal dialysis patients.

\subsection{Media and growth conditions}

Tryptic soy broth (TSB) and tryptic soy agar (TSA) were prepared according to the manufacturer's instructions. All strains were incubated in $15 \mathrm{ml}$ of TSB inoculated with bacteria grown on TSA plates not older than 2 days, and grown for $24( \pm 2) \mathrm{h}$ at $37^{\circ} \mathrm{C}$ in a shaker rotator at $130 \mathrm{rpm}$. Then, $200 \mu \mathrm{l}$ of each cell suspension was transferred to $150 \mathrm{ml}$ of fresh TSB, which was incubated for $18( \pm 2)$ h at $37^{\circ} \mathrm{C}$ at $130 \mathrm{rpm}$. After being harvested by centrifugation (for $5 \mathrm{~min}$ at $10500 \mathrm{~g}$ and $4{ }^{\circ} \mathrm{C}$ ), cells were washed twice and resuspended in a saline solution $(0.9 \% \mathrm{NaCl}$ prepared in distilled water).

\subsection{Substrate preparation}

Acrylic was cut into $20 \times 20 \mathrm{~mm}$ squares (for static method) or into $76 \times 50 \mathrm{~mm}$ plates (for dynamic method). These substrata were immersed in a $0.2 \%$ solution of a commercial detergent overnight, after which they were transferred to a new solution of $0.2 \%$ of a commercial detergent and washed at $40{ }^{\circ} \mathrm{C}$ with strong agitation for $5 \mathrm{~min}$. The squares and plates were then well rinsed with distilled water and finally each individual substratum was well rinsed with ultra-pure water and dried at $60^{\circ} \mathrm{C}$, overnight.

\subsection{Static adhesion methods}

\subsubsection{Optimization of the adhesion assays}

In order to ascertain the influence of the initial inoculum upon the numbers of adhering cells, adhesion was allowed to occur for $30 \mathrm{~min}$ using different cell concentrations. In order to determine the influence of the adhesion time on the amount of adherent bacterial cells, adhesion was allowed to occur for different times, using a standard cell concentration.

To evaluate the effect of the washing step, 3 approaches were considered: no washing, washing by multiple immersions in water and finally washing by rinsing with a spurt of water. When using the immersion method, the acrylic surfaces were gently transferred to $100 \mathrm{ml}$ glass beakers containing distilled water, and were allowed to rest there for approximately $10 \mathrm{~s}$. Afterwards, a new transfer was made to a different $100 \mathrm{ml}$ glass beaker with distilled water, followed by a third transfer $10 \mathrm{~s}$ later. In the other method, the substrate surfaces were carefully removed from the adhesion medium and were rinsed with a spurt of distilled water for approximately $20 \mathrm{~s}$. After the washing steps, all surfaces were allowed to dry at $60^{\circ} \mathrm{C}$.

S. epidermidis 9142 was selected as the strain to be used in all the optimization assays, since it is a known strain already used in other works $[15,16]$.

\subsubsection{Static adhesion}

Squares of acrylic were placed in 6 well tissue-culture plates containing $5 \mathrm{ml}$ of a cell suspension at an optimal concentration in saline solution (see Table 1). Initial adhesion to each substratum was allowed to occur during the optimal adhesion time (see Table 1) at $37^{\circ} \mathrm{C}$, in a shaker at $120 \mathrm{rpm}$. Negative controls were obtained by placing acrylic in a saline solution without bacterial cells. The squares were then carefully washed by immersion. The substratum squares with adhered cells were dried at $37^{\circ} \mathrm{C}$. All experiments were done in triplicate, with 4 repeats.

\subsubsection{Image analysis}

For image observation and enumeration of adherent bacterial cells, the substratum squares were stained with a $0.2 \%$ safranin solution for better contrast. Direct bacterial counts were done using a phase contrast microscope coupled to a 
$3 C C D$ video camera that acquires images with $820 \times 560$ pixels resolution at a magnification of $400 \times$. With this magnification $1 \mathrm{~cm}^{2}$ is equivalent to $1.823 \times 10^{4}$ captured images (as determined by a Neubauer chamber). For each surface analyzed, 20 images were taken. Cells were counted using automated enumeration software.

\subsection{Dynamic adhesion methods}

\subsubsection{The parallel-plate flow chamber and image analysis}

The parallel-plate flow chamber and image analysis system has been previously described [5]. Briefly, images were taken from the center of the bottom of the plate, with a $3 \mathrm{CCD}$ video camera mounted on a phase-contrast inverted microscope equipped with a $40 \times$ ultra-long working distance objective. Images containing $768 \times 576$ pixels were acquired by image analysis software. Every $10 \mathrm{~s}$ one image was obtained by adding 5 consecutive images captured with a time interval of $500 \mathrm{~ms}$ in order to eliminate moving bacteria and to enhance the signal-to-noise ratio. At the magnification used, $1 \mathrm{~cm}^{2}$ is equivalent to $3.148 \times 10^{4}$ captured images (as determined with a Neubauer chamber).

\subsubsection{Dynamic adhesion}

Prior to each experiment, all tubes and the flow chamber were filled with saline solution with special attention to removing all air bubbles from the system. A saline solution was circulated through the system until the stationary operation conditions were obtained $(0.18 \pm 0.02 \mathrm{ml} / \mathrm{s})$, which yields a laminar flow (Reynolds number of $4.8 \pm 0.6$ ). A pulse-free flow was established by hydrostatic pressure, and the suspension was recirculated using a peristaltic roller pump.

Afterwards, the flow was switched to the bacterial suspension (at the same concentration used in the static assays) that was circulated throughout the system at room temperature for $60 \mathrm{~min}$ while images were captured with a time interval of $10 \mathrm{~s}$. After the enumeration of adhering bacteria, an air-liquid interface was passed through the system and the amount of cells removed was determined. In addition to the determination of adherent bacteria at a stationary endpoint, the initial deposition rate was also calculated during the first min of the adhesion assays. All experiments were done at least 4 times with independent cultures.

\subsection{Statistical analysis}

All the adherence assays were compared using oneway analysis of variance (ANOVA) by applying Levene's test of homogeneity of variances, and the Tukey multiplecomparisons test, and also the paired samples $t$-test using SPSS software (Statistical Package for the Social Sciences). All tests were performed with a confidence level of $95 \%$.

\section{Results}

\subsection{Optimization of the adhesion assays}

A summary of the optimization of the static adhesion parameters is presented in Table 1.

When no washing step was performed, the number of adherent cells was found to be constant at different adhesion times. On the other hand, when using immersion or rinsing washing procedures, the number of adherent cells increased with increasing adhesion times. However, only in washing by immersion was this increase found to be significantly different at the $P<0.05$ level (ANOVA and Tukey's multiple-comparison test).

As expected, a lower initial cellular concentration yielded a lower number of adherent organisms but interestingly the differences in adherent organisms over a broad range of initial inocula were not large. Clearly there is a maximum number of bacterial cells that can adhere to the substrate, as shown by the similar level of adhesion achieved by a cell suspension with an initial optical density of 1 compared with another suspension with an initial optical density of 2.4.

When comparing the number of adherent cells after different exposure times, we verified that lower exposure times yielded a lower level of adherent cells $\left(2.3 \times 10^{6}\right.$ cells $/ \mathrm{cm}^{2}$ after $30 \mathrm{~min}$ of exposure and $4.2 \times 10^{6}$ cells $/ \mathrm{cm}^{2}$ after $120 \mathrm{~min}$ of exposure). The plateau was attained at $120 \mathrm{~min}$ of exposure, as there was no significant increase in adherence at the $P<0.05$ level (ANOVA and Tukey's multiplecomparison test) after $150 \mathrm{~min}$ of exposure $\left(4.6 \times 10^{6}\right.$ and $4.5 \times 10^{6}$ cells $/ \mathrm{cm}^{2}$, respectively).

\subsection{Comparison of static with dynamic adhesion}

A summary of the adhesion parameters using the two methods is presented in Table 2. A linear relation was obtained between the adhesion levels (in $10^{6} \mathrm{cell} / \mathrm{cm}^{-2}$ ) in dynamic method $(D)$ and static method $(S): D=3.4011 \times S+$ $5.6288, r=0.9555$. When using the static method the only information that can be obtained is the amount of cells adherent at the end of the assay. When using a dynamic method, more information is available. As can be seen in Fig. 1, the 3 strains, representative of the distinct behaviors observed, reached a maximal adhesion phase very quickly (in less than 30 min) compared with 120 min using the static method. Furthermore, the number of adherent bacterial cells with the dynamic method was almost 10 -fold higher than the adhesion achieved in the static method $(P<0.05$, paired samples $t$-test). The results obtained by the static adhesion method revealed that $S$. epidermidis strains adhered at higher extents to acrylic than $S$. haemolyticus strains (Table 2). The only exception was $S$. epidermidis IE75 that adhered at the same level as S. haemolyticus IE246 and S. haemolyticus M176 $\left(2.2 \times 10^{6}, 2.6 \times 10^{6}\right.$ and $3.1 \times 10^{6}$ cells $/ \mathrm{cm}^{2}$, respectively) . With the dynamic adhesion assay, the extent of adherence of 
Table 1

Optimization of the static adhesion parameters

\begin{tabular}{|c|c|c|c|c|c|c|}
\hline Adhesion time $^{\mathrm{a}}$ (min) & $10^{6}$ cell $/ \mathrm{cm}^{-2}$ & Initial inoculum $^{\mathrm{b}}$ & $10^{6}$ cell $/ \mathrm{cm}^{-2}$ & Washing step ${ }^{c}$ & Adhesion time (min) & $10^{6} \mathrm{cell} / \mathrm{cm}^{-2}$ \\
\hline 15 & $1.2( \pm 0.3)$ & 0.1 & $0.3( \pm 0.2)$ & \multirow[t]{2}{*}{ None } & 20 & $35.2( \pm 5.7)$ \\
\hline 30 & $2.3( \pm 0.2)$ & 0.4 & $0.7( \pm 0.2)$ & & 40 & $36.4( \pm 9.9)$ \\
\hline 60 & $3.6( \pm 0.4)^{*}$ & $1.0^{\#}$ & $1.3( \pm 0.2)^{*}$ & \multirow[t]{2}{*}{ Immersion } & 20 & $1.2( \pm 0.3)^{*}$ \\
\hline 120 & $4.6( \pm 0.5)$ & $1.8^{\#}$ & $2.1( \pm 0.2)$ & & 40 & $2.3( \pm 0.4)^{*}$ \\
\hline \multirow[t]{2}{*}{180} & $4.6( \pm 0.4)$ & $2.4^{\#}$ & $2.2( \pm 0.3)$ & \multirow[t]{2}{*}{ Rinsing } & 20 & $0.6( \pm 0.5)$ \\
\hline & & & & & 40 & $0.9( \pm 0.5)$ \\
\hline
\end{tabular}

* Represents a mean difference significant at the $P<0.05$ level (ANOVA and Tukey's multiple-comparison test).

${ }^{\text {a }}$ Influence of the adhesion time upon the number of adhering cells. A standard inoculum was used with an optical density of 2.0 (diluted 4-fold, measured 0.5 at $640 \mathrm{~nm})$.

b Influence of the initial cell density upon the number of adhering cells (cell densities described above $0.8\left({ }^{\#}\right)$ were diluted 4 -fold and measured at 640 nm).

c Influence of the washing steps at different times of adhesion upon the numbers of adhering cells. The standard deviation is between brackets.

most of the $S$. epidermidis strains was again significantly different ( $P<0.05$, ANOVA and Tukey's multiple-comparison test) from the $S$. haemolyticus strains, when comparing the adhesion achieved at the stationary phase. Regarding the comparison of the initial adhesion rates, S. haemolyticus strains had the slowest rates, significantly different from the rates of the $S$. epidermidis strains $(P<0.05$, ANOVA and Tukey's multiple-comparison test). The effect of passing an air-bubble through adherent cells in the parallel plate flow chamber resulted in removal of a large proportion of the adherent organisms. This can be explained by the formation of an air-liquid interface, that induces a shear force of about $10^{-7} \mathrm{~N}$, which is in the range of the adhesion strength evaluated by atomic force microscopy [11].

\section{Discussion}

Many investigators have evaluated the adherence of bacteria such as CoNS to polymers and medical devices to understand the processes of colonization and biofilm formation $[1,13,21]$. However, comparisons of parameters that might be important in evaluating the results achieved, such as the amount of inoculum, incubation period and washing procedure, are not fully discussed in the literature. Additionally, there is a lack of detailed studies comparing both the static and the dynamic methods of assessing initial adhesion. In this study we evaluated several critical parameters that could affect the outcome of adherence assays to provide insights into which steps of this process are affected by variations in experimental conditions.

In the present case, when no washing step was used, a high number of adherent bacterial cells were obtained. Furthermore, without washing, there was no difference between levels of adherent cells regardless of the exposure times to the substratum. It is probable that the cells enumerated include those that adhered, plus the loosely attached cells and the cells deposited by sedimentation. While it might seem obvious that some washing must occur to discriminate effectively adherent from merely deposited cells, the findings without washing point out the need for defining a proper set of washing conditions to accurately gauge and compare

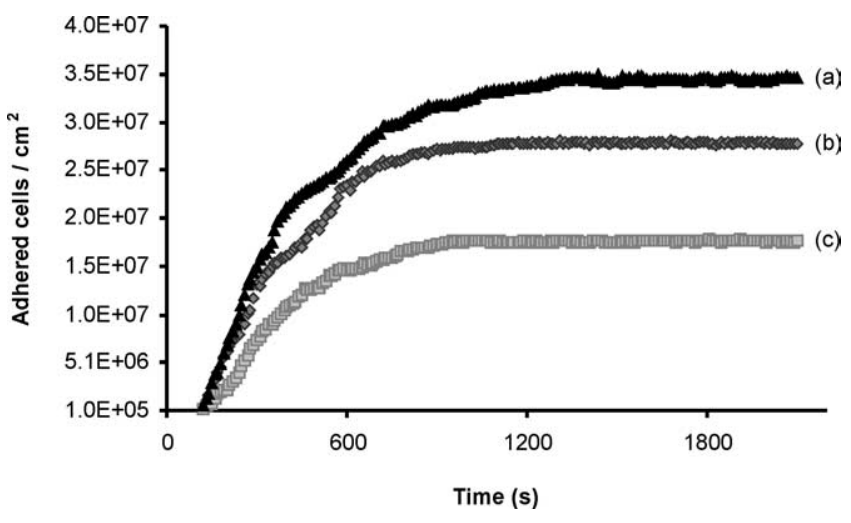

Fig. 1. Adherence kinetics of S. epidermidis 9142 (a), S. epidermidis IE75 (b) and S. haemolyticus M176 (c) using the dynamic parallel flow chamber assay.

among different experimental conditions the number of adherent cells on a substratum.

When further comparing the two distinct washing procedures, both of which are referred to in the literature [2,3,17], it was found that adhesion levels determined by the two methods were different for each time of exposure evaluated, demonstrating the importance of choosing an adequate method to remove non-adherent cells when using static adhesion assays. Moreover, both methods revealed an increase in the number of adherent cells with increasing time of exposure. Washing by immersion was considered to be more reliable since it is more reproducible. Moreover, the spurt method is subjected to variations dependent on the operator since the amount and flow of water used will probably vary between repeated experiments. Based on methodological investigations, we used the static adherence assays along with the immersion method of washing the surfaces to compare bacterial adherence after $2 \mathrm{~h}$, a time found to yield maximal adherence. The initial cell suspension had a concentration equivalent to $1 \times 10^{9}$ cells $/ \mathrm{ml}$, which was found to be the "breakthrough concentration", in the sense that it corresponds to the highest level of adherence with the lowest input inoculum.

A clear difference was found when comparing the extent of adhesion of $S$. epidermidis with S. haemolyticus, 
Table 2

CONS adhesion to acrylic in both dynamic and static methods

\begin{tabular}{|c|c|c|c|c|}
\hline \multirow[t]{2}{*}{ Strain } & \multicolumn{3}{|c|}{ Dynamic method } & \multirow{2}{*}{$\frac{\text { Static method }}{n_{120 \min ^{\mathrm{d}}}{ }^{\mathrm{n}}}$} \\
\hline & $j_{0}{ }^{\mathrm{a}}$ & $n_{30 \text { min }}{ }^{\mathrm{b}}$ & $\mathrm{d} \%^{\mathrm{c}}$ & \\
\hline S. epidermidis 9142 & $7.2( \pm 1.2)$ & $36.8( \pm 7.4)^{*}$ & $91( \pm 5)$ & $4.5( \pm 0.5)$ \\
\hline S. epidermidis IE75 & $5.9( \pm 0.9)$ & $23.4( \pm 5.1)$ & $87( \pm 12)$ & $2.2( \pm 0.7)$ \\
\hline S. epidermidis IE186 & $12.2( \pm 0.9)$ & $44.3( \pm 4.0)^{*}$ & $80( \pm 12)$ & $5.2( \pm 1.9)$ \\
\hline S. epidermidis M187 & $10.7( \pm 0.2)$ & $40.4( \pm 5.9)^{*}$ & $79( \pm 3)$ & $4.6( \pm 1.4)$ \\
\hline S. haemolyticus M176 & $3.3( \pm 0.6)^{*}$ & $17.1( \pm 1.9)$ & $91( \pm 7)$ & $3.1( \pm 1.1)$ \\
\hline
\end{tabular}

* Represents a mean difference significant at the $P<0.05$ level (ANOVA and Tukey's multiple-comparison test).

a Initial adhesion rate $\left(j_{0}, 10^{4}\right.$ cell $\left./ \mathrm{cm}^{2} \mathrm{~s}^{1}\right)$.

b Number of adhering bacteria at stationary deposition phase $\left(n_{30 \mathrm{~min}}, 10^{6} \mathrm{cell} / \mathrm{cm}^{-2}\right)$.

c Percentage of bacteria detached by passing an air-bubble through the chamber.

$\mathrm{d}$ Number of adhering bacteria at stationary deposition phase $\left(n_{120 \mathrm{~min}}, 10^{6} \mathrm{~cm}^{2}\right)$. The standard deviation is between brackets

with most $S$. epidermidis adhering to a greater extent than $S$. haemolyticus strains. This was also seen in the 3 parameters measured with the dynamic method: generally, $S$. epidermidis had a higher initial rate of adherence and reached higher cellular densities in the stationary phase of adhesion which was also attained sooner; and they were more resistant to removal after passing an air-bubble through the parallel plate flow chamber. Although the static method allows only one parameter to be measured (the number of adhering cells at the stationary point), it was also found that $S$. epidermidis generally adheres better to acrylic than S. haemolyticus. Because of this, two hierarchal cluster analyses, based on squared Euclidean distances, were performed as described by Bosch et al. [23] using the data from static and from dynamic adhesion assays. From both cluster analyses (Fig. 2), two distinct groups could be identified: both $S$. haemolyticus and S. epidermidis IE75 belong to the same group, showing the lowest extent of adhesion; the remaining S. epidermidis strains belong to another distinct group, displaying the greatest number of adhering cells.

With the dynamic method, adherence was almost 10-fold higher than that achieved with the static method. This can be explained by the differences in the transport phenomena in the two methods. With the static method, transport of bacteria is by diffusion only, while with the dynamic method, in addition to diffusion, there are also convection phenomena [19] that could contribute to bacterial accumulation. It has been demonstrated previously that the transport phenomena involved in adhesion processes greatly influence the extent of adhesion [4].

It has been suggested that, since the slide method will always be affected by the air-liquid interface in the washing and drying processes, this method evaluates the retention of cells rather than adhesion [5], i.e., the ability to adhere to a surface and to resist shear forces that exist in many natural environments $[6,8,9]$. However, the effect of the air-liquid interface varies and is dependent on the substratum properties, cell surface properties and the velocity of the passing air-bubble $[7,12]$. In this study, the washing procedure was (a)

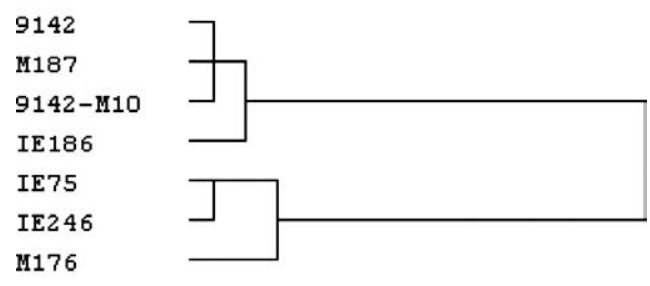

(b)

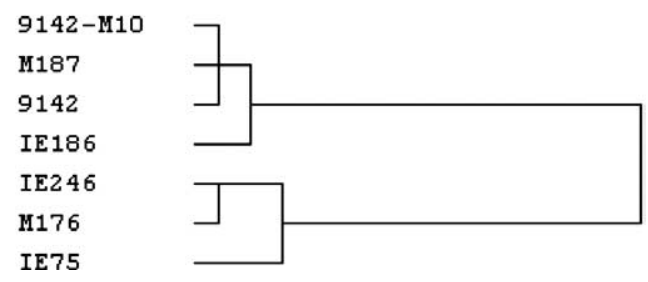

Fig. 2. Hierarchal Cluster analysis based on squared Euclidean distances of (a) static adhesion assays and (b) dynamic adhesion assays.

performed carefully in order to avoid the formation of an air-liquid interface.

Overall, the results obtained in the present work suggest that both static and dynamic methods are equally valid to compare adhesion behaviors among CoNS clinical strains. The linear relation obtained between adhesion levels in the dynamic method and the static method had a very high correlation. Additionally, the cluster analysis performed using either static or dynamic adhesion results yielded the same two distinct groups, differentiating between the strains by their adhesion capabilities. For instance, the high capacity of adhesion of $S$. epidermidis IE186 compared to any of the $S$. haemolyticus can be detected by both static and dynamic methods. Although dynamic method do provide more information, the ease and practicality of a well-defined static method can also be useful for discerning differences among CoNS strains in terms of their adherence ability. 
In summary, the static adhesion method can be useful for evaluating differences in adhesion capabilities, even if it only provides information about the final level of adherence achieved. Since it is a low cost method that is very easy to perform and less-time consuming, the slide static adherence method has some advantages over the dynamic parallel-plate flow chamber method, such as evaluating the adherence capacity of a high number of strains. The dynamic adhesion assay using the parallel-plate flow chamber is more time-consuming and needs more expensive equipment, and is thus more appropriate for studying fundamental aspects of adhesion with a limited number of strains, as has been demonstrated by many authors [12,18,22].

\section{Acknowledgements}

The authors acknowledge the financial support of FCT, through the project FCT POCTI/ESP/42688/2001, and also grant SFRH/BD/8676/2002. GBP was supported by NIH grant AI 46706.

\section{References}

[1] H. Akiyama, O. Yamasaki, H. Kanzaki, J. Tada, J. Arata, Adherence characteristics of Staphylococcus aureus and coagulase-negative staphylococci isolated from various skin lesions, J. Dermatol. Sci. 18 (1998) 132-136.

[2] Y. An, R. Friedman, Laboratory methods for studies of bacterial adhesion, J. Microbiol. Meth. 30 (1997) 141-152.

[3] J. Azeredo, R. Oliveira, The role of exopolymers in the attachment of Sphingomonas paucimobilis, Biofouling 61 (2000) 59-67.

[4] D. Bakker, H. Busscher, H. Van der Mei, Bacterial deposition in a parallel plate and a stagnation point flow chamber: Microbial adhesion mechanisms depend on the mass transport conditions, Microbiology 148 (2002) 597-603.

[5] R. Bos, H. Van der Mei, H. Busscher, Physico-chemistry of initial microbial adhesive interactions - its mechanisms and methods for study, FEMS Microbiol. Rev. 23 (1999) 179-230.

[6] G. Bruinsma, H. Van der Mei, H. Bussher, Bacterial adhesion to surface hydrophilic and hydrophobic contact lenses, Biomaterials 22 (2001) 3217-3224.

[7] H. Busscher, A. Weerkamp, H. Van der Mei, A. Van Pelt, H. Jong, J. Arends, Measurement of the surface free-energy of bacterial-cell surfaces and its relevance for adhesion, Appl. Environ. Microb. 48 (1984) 980-983.

[8] H. Busscher, R. Bos, H. van der Mei, Initial microbial adhesion is a determinant for the strength of biofilm adhesion, FEMS Microbiol. Lett. 128 (1995) 229-234.
[9] H. Busscher, G. Geertsema-Doornbusch, H. van der Mei, Adhesion to silicone rubber of yeast and bacteria isolated from voice prostheses: Influence of salivary conditioning films, J. Biom. Mat. Res. 34 (1997) 201-210.

[10] J. Dankert, A. Hogt, J. Feijen, Biomedical polymers: Bacterial adhesion, colonization and infection, CRC Critic. Rev. Biocompat. 2 (1986) 219-301.

[11] H. Fang, K. Chan, L. Xu, Quantification of bacterial adhesion forces using atomic force microscopy (AFM), J. Microbiol. Meth. 40 (2000) 89-97.

[12] C. Gómez-Suárez, H. Busscher, H. Van der Mei, Analysis of bacterial detachment from substratum surfaces by the passage of air-liquid interfaces, Appl. Environ. Microb. 67 (2001) 2531-2537.

[13] M. Gross, S. Cramnton, F. Gotz, A. Peschel, Key role of teichoic acid net charge in Staphylococcus aureus colonization of artificial surfaces, Infect. Immun. 69 (2001) 3423-3426.

[14] F. Lalla, Antimicrobial chemotherapy in the control of surgical infectious complications, J. Chemotherapy 11 (1999) 440-445.

[15] D. Mack, J. Riedewald, H. Rohde, T. Magnus, H. Feutcht, H. Elsner, R. Laufs, M. Rupp, Essential functional role of the polysaccharide intercellular adhesin of Staphylococcus epidermidis in hemagglutination, Infec. Immun. 67 (1999) 1004-1008.

[16] D. Mack, H. Rohde, S. Dobinsky, J. Riedewald, M. Nedelmann, J. Knobloch, H. Elsner, H. Feutch, Identification of three essential regulatory gene loci governing expression of Staphylococcus epidermidis polysaccharide intercellular adhesin and biofilm formation, Infec. Immun. 68 (2000) 3799-3807.

[17] D. McKenney, J. Hubner, E. Muller, Y. Wang, D. Goldmann, G. Pier, The ica locus of Staphylococcus epidermidis encodes production of the capsular polysaccharide/Adhesin, Infect. Immun. 66 (1998) 47114720.

[18] K. Millsap, G. Reid, H. Van der Mei, H. Busscher, Adhesion of Lactobacillus species in urine and phosphate buffer to silicone rubber and glass under flow, Biomaterials 18 (1996) 87-91.

[19] N. Mohamed, M. Teeters, J. Patti, M. Hook, J. Ross, Inhibition of Staphylococcus aureus adherence to collagen under dynamic conditions, Infect. Immun. 67 (1999) 589-594.

[20] J. O’Gara, H. Humphreys, Staphylococcus epidermidis biofilms: Importance and implications, J. Med. Microb. 50 (2001) 582-587.

[21] E. Ostuni, R. Chapman, M. Liang, G. Meluleni, G. Pier, D. Ingber, G. Whitesides, Self-assembled monolayers that resist the adsorption of proteins and the adhesion of bacterial and mammalian cells, Langmuir 17 (2001) 6336-6343.

[22] H. Rijnaarts, W. Norde, E. Bouwer, J. Lyklema, A. Zehnder, Bacterial adhesion under static and dynamic conditions, Appl. Environ. Microb. 59 (1993) 3255-3265.

[23] J. Bosch, H. van der Mei, H. Busscher, Statistical analysis of bacterial species based on a physico-chemical surface proteins, Biofouling 4 (1991) 141-150.

[24] H. Van der Mei, B. Van de Belt-Gritter, G. Reid, H. BialkowskaHobrzanska, H. Busscher, Adhesion of coagulase-negative staphylococci grouped according to physico-chemical surface properties, Microbiology 143 (1997) 3861-3870.

[25] C. Voung, M. Otto, Staphylococcus epidermidis infections, Microbes Infect. 4 (2002) 481-489. 